\title{
Efficient Acyclic Stereocontrol Using the Tethered Aminohydroxylation \\ (TA) Reaction
}

\author{
Timothy J. Donohoe*, Peter D. Johnson, Richard J. Pye, \\ and Martine Keenan
}

\section{Supplementary Material}

\section{General Experimental Procedures}

Preparation of tert-Butyl hypochlorite ${ }^{1}$ (prepared in the dark)

A $4 \%$ aqueous solution of sodium hypochlorite was prepared by diluting sodium hypochlorite (100 mL, 12\% available chlorine, supplied by BDH Chemicals) in deionised water $(150 \mathrm{~mL})$ and the resulting solution was cooled to $0^{\circ} \mathrm{C}$. To this was added a solution of glacial acetic acid $(12 \mathrm{~mL})$ and tert-butanol $(18 \mathrm{~mL})$ in one portion and the resulting mixture was stirred for 3 mins. The organic layer was separated and washed successively with sodium carbonate $(10 \%$ solution, $25 \mathrm{~mL})$ and water $(25 \mathrm{~mL})$. The product was dried over calcium chloride and filtered to yield the title compound as a yellow liquid. The product was stored below $4^{\circ} \mathrm{C}$ in the dark over calcium chloride.

\section{General Carbamate Formation Procedure ${ }^{2}$}

Trichloroacetyl isocyanate $(1.2 \mathrm{mmol})$ was added dropwise to a solution of the alcohol (1 $\mathrm{mmol})$ in dry dichloromethane $(1.5 \mathrm{~mL} / \mathrm{mmol})$ at $0^{\circ} \mathrm{C}$. After stirring for $2 \mathrm{~h}$, or until TLC showed no starting material present, the mixture was concentrated under reduced 
pressure. The residue was dissolved in methanol $(2 \mathrm{~mL} / \mathrm{mmol})$, cooled to $0^{\circ} \mathrm{C}$ and an aqueous potassium carbonate solution $(3 \mathrm{mmol}, 2 \mathrm{~mL} / \mathrm{mmol})$ was added. The cooling bath was removed and the mixture was allowed to stir for $4 \mathrm{~h}$, by which time TLC showed complete conversion. The methanol was evaporated under reduced pressure and the aqueous residue was extracted with dichloromethane $(25 \mathrm{~mL}$ x 3). The combined organics were washed with brine $(10 \mathrm{~mL})$, dried over magnesium sulfate and concentrated under reduced pressure to yield the crude carbamate, which was purified by flash column chromatography on silica or recrystallisation if necessary.

\section{General Tethered Aminohydroxylation Procedure}

A fresh aqueous solution of sodium hydroxide $(0.08 \mathrm{M}, 0.9$ eq.) was prepared. All but a few drops of this was added in one portion to a stirred solution of the allylic carbamate (1.0 eq.) in propan-1-ol $(12 \mathrm{~mL} / \mathrm{mmol})$. The solution was allowed to stir for $5 \mathrm{mins}$, before freshly prepared tert-butyl hypochlorite (1 eq.) was added. The mixture was again allowed to stir for 5 mins. To this was added diisopropyl ethylamine (5 mol\%) in one portion. The mixture was allowed to stir for a further 5 mins before the final addition of a solution of potassium osmate $(4 \mathrm{~mol} \%)$ in the remainder of the sodium hydroxide solution made earlier. The reaction was monitored by TLC and halted when no further change was detected. The reaction was quenched by the addition of sodium sulfite (500 $\mathrm{mg}$ ), and allowed to stir for 30 mins. The mixture was extracted with ethyl acetate (x 5). The combined organics were washed with brine, dried over sodium sulfate and concentrated under reduced pressure. The crude material was purified by flash column chromatography on silica. 


\section{Spectroscopic Data}

The minor compounds present in the NMR spectra of compounds $2,4,6,10,12$ and 18 is the anti diastereosiomer of product.

\section{(2E)-1-Propyl-hex-2-enyl carbamate (1)}

$(5 E)-N o n e n-4-\mathrm{ol}^{3}(770 \mathrm{mg}, 5.4 \mathrm{mmol})$ was subjected to the general carbamate formation procedure, affording the carbamate 1 as a yellow solid (960 mg, 95\%).

$R_{\mathrm{f}}(\mathrm{EtOAc}) 0.69 ;$ m.p. $29-32{ }^{\circ} \mathrm{C}$; IR $v_{\max }\left(\mathrm{CHCl}_{3}\right) 3453,3344\left(\mathrm{NH}_{2}\right), 1706(\mathrm{C}=\mathrm{O})$ and 1601 $\left(\mathrm{NH}_{2}\right) ;{ }^{1} \mathrm{H}$ NMR $\delta_{\mathrm{H}}\left(400 \mathrm{MHz} ; \mathrm{CDCl}_{3}\right) 5.71(1 \mathrm{H}, \mathrm{dt}, J 15.0$ and 7.0, CHPr $), 5.39(1 \mathrm{H}$, ddt, $J$ 15.0, 8.0 and 2.0, CH=CHPr), $5.08(1 \mathrm{H}, \mathrm{q}, J$ 7.0, CHOCO $), 4.65\left(2 \mathrm{H}, \mathrm{br} \mathrm{s}, \mathrm{NH}_{2}\right)$, 2.04-1.99 (2 H, m, allylic $\left.\mathrm{CH}_{2}\right), 1.66-1.54\left(2 \mathrm{H}, \mathrm{m}, \mathrm{CHOCH}_{2}\right), 1.43-1.31(4 \mathrm{H}, \mathrm{m}, 2 \times$ $\left.\mathrm{MeCH}_{2}\right)$ and 0.95-0.87 (6 H, m, $\left.2 \times \mathrm{Me}\right) ;{ }^{13} \mathrm{C}$ NMR $\delta_{\mathrm{C}}\left(\mathrm{CDCl}_{3}\right) 156.7(\mathrm{C}=\mathrm{O}), 133.8$ $\left(C_{\mathrm{A}}=\mathrm{C}_{\mathrm{B}}\right), 128.7\left(\mathrm{C}_{\mathrm{A}}=C_{\mathrm{B}}\right), 75.6(\mathrm{CHOR}), 36.8\left(\mathrm{CH}_{2} \mathrm{CHOR}\right), 34.3,22.1,18.4\left(3 \times \mathrm{CH}_{2}\right)$, 13.9 and $13.6\left(2 \times \mathrm{CH}_{3}\right)$; MS m/z (CI) $186\left(\mathrm{MH}^{+}\right), 142\left(\mathrm{MH}^{+}-\mathrm{CONH}_{2}\right), 125\left(100 \%, \mathrm{MH}^{+}\right.$ - $\mathrm{OCONH}_{2}$ ) and 95; HRMS(CI) Found $\mathrm{MH}^{+}, 186.1496 . \mathrm{C}_{10} \mathrm{H}_{20} \mathrm{NO}_{2}$ requires $M$ 186.1494.

\section{(1'SR,4RS,5RS)-4-(1'-Hydroxybutyl)-5-propyloxazolidin-2-one (2)}

Prepared from (2E)-1-propyl-hexenyl carbamate (1) via the general TA procedure. The crude material was purified by flash column chromatography on silica, eluting with ethyl acetate $(100 \%)$, to furnish the title compound as a white solid (120 mg, 57\%, 6:1); m.p. $87-90{ }^{\circ} \mathrm{C}$; IR $v_{\max } \mathrm{cm}^{-1}\left(\mathrm{CHCl}_{3}\right) 3305,2960,2874,1747,1731 ;{ }^{1} \mathrm{H}$ NMR $\delta_{\mathrm{H}}(400 \mathrm{MHz}$, $\left.\mathrm{CDCl}_{3}\right)$ (major isomer) $6.48(1 \mathrm{H}$, br s, $\mathrm{NH}), 4.39(1 \mathrm{H}, \mathrm{dt}, J 8.5$ and $J$ ' 5.0, CHOCO), 3.46

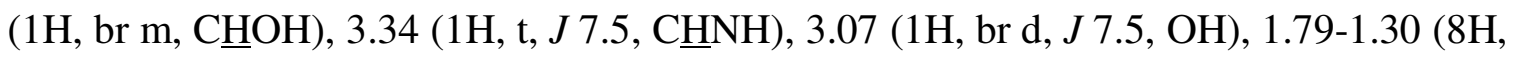


m, $\left.2 \mathrm{xCH}_{2} \mathrm{CH}_{2}\right), 1.00-0.93\left(6 \mathrm{H}, \mathrm{m}, 2 \mathrm{xCH}_{3}\right) ;{ }^{13} \mathrm{C} \mathrm{NMR} \delta_{\mathrm{C}}\left(100 \mathrm{MHz}, \mathrm{CDCl}_{3}\right)$ 160.4, 79.4, $72.1,62.7,37.2,35.2,18.9,17.9,13.9,13.7 ; \mathrm{m} / \mathrm{z}(\mathrm{CI}) 219\left(\mathrm{MNH}_{4}^{+}\right)$and $202(100 \%$, $\mathrm{MH}^{+}$); HRMS for $\mathrm{C}_{10} \mathrm{H}_{23} \mathrm{~N}_{2} \mathrm{O}_{3}$ requires 219.1709, found 219.1712.

\section{4-(tert-Butyldiphenylsilanyloxy)-1-methyl-but-2E-enyl carbamate (3)}

Carbamate (3) was prepared from 6-(tert-butyldiphenylsilanyloxy)-hex-4-en-2-ol ${ }^{4}$ following the standard carbamation procedure to furnish the title compound as a waxy solid (291 mg, 87\%); ${ }^{1} \mathrm{H}$ NMR $\delta_{\mathrm{H}}\left(200 \mathrm{MHz}, \mathrm{CDCl}_{3}\right)$ 7.71-7.67 (4H, m, Ph), 7.45-7.36 $(6 \mathrm{H}, \quad \mathrm{m}, \mathrm{Ph}), \quad 5.81-5.77 \quad\left(2 \mathrm{H}, \quad \mathrm{m}, \quad \mathrm{TBDPSOCH}_{2} \mathrm{C} \underline{\mathrm{H}}=\mathrm{C} \underline{\mathrm{H}}\right), \quad 5.34-5.26 \quad(1 \mathrm{H}, \mathrm{m}$, TBDPSOCH $\left.\mathrm{C}_{2} \mathrm{CH}=\mathrm{CH}\right), 4.78\left(2 \mathrm{H}, \mathrm{br} \mathrm{s}, \mathrm{NH}_{2}\right), 4.22-4.21\left(2 \mathrm{H}, \mathrm{m}, \mathrm{CH}_{2} \mathrm{OTBDPS}\right), 1.33(3 \mathrm{H}$, d, $J$ 6.5, $\left.\mathrm{CH}_{3}\right), 1.08\left(9 \mathrm{H}, \mathrm{s},\left(\mathrm{CH}_{3}\right)_{3}\right) ;{ }^{13} \mathrm{C} \mathrm{NMR} \delta_{\mathrm{C}}\left(50 \mathrm{MHz}, \mathrm{CDCl}_{3}\right) 156.9,136.0,134.0$, $131.1,130.1,129.9,128.1,71.7,64.1,27.3,20.9,19.7 ; \mathrm{m} / \mathrm{z}$ (ESI) $442(100 \%$ $\mathrm{M}+\mathrm{NH}_{4}^{+}+\mathrm{CH}_{3} \mathrm{CN}$ ); $\mathrm{HRMS}$ for $\mathrm{C}_{22} \mathrm{H}_{29} \mathrm{NO}_{3} \mathrm{NaSi}$ requires 406.1814, found 406.1804.

\section{(1'S,4S,5S)-4-[2'-(tert-Butyldiphenylsilanyloxy)-1'-hydroxy-ethyl]-5-}

\section{methyloxazolidin-2-one (4)}

Prepared from 4-(tert-butyldiphenylsilanyloxy)-1-methyl-but-2E-enyl carbamate (3) via the general TA procedure. The crude material was purified by flash column chromatography on silica, eluting with $40-60^{\circ} \mathrm{C}$ petrol:ethyl acetate (1:1), to furnish the title compound as a colourless oil $(194 \mathrm{mg}, 66 \%, 5: 1) ;[\alpha]^{20}{ }_{\mathrm{D}}+25.8\left(\right.$ c. $\left.1.00, \mathrm{CHCl}_{3}\right)$; IR $v_{\text {max }} \mathrm{cm}^{-1}\left(\mathrm{CHCl}_{3}\right) 3410,3071,2930,2857,1742 ;{ }^{1} \mathrm{H} \mathrm{NMR} \delta_{\mathrm{H}}\left(400 \mathrm{MHz}, \mathrm{CDCl}_{3}\right.$ ) (major isomer) 7.67-7.63 (4H, m, Ph), 7.47-7.39 (6H, m, Ph), $6.08(1 \mathrm{H}, \mathrm{br}, \mathrm{NH}), 4.50(1 \mathrm{H}, \mathrm{dq}$, 
$J$ 6.4, CHOCO), 3.68 (2H, d, $J$ 4.6, $\left.\mathrm{C}_{2} \mathrm{OTBDPS}\right), 3.59-3.51(2 \mathrm{H}, \mathrm{m}, \mathrm{C} \underline{\mathrm{HNH}}$ and $\mathrm{C} \underline{\mathrm{HOH}}), 3.34\left(1 \mathrm{H}, \mathrm{d}, J\right.$ 7.1, OH), $1.36\left(3 \mathrm{H}, \mathrm{d}, J 6.3, \mathrm{CH}_{3}\right), 1.08\left(9 \mathrm{H}, \mathrm{s},\left(\mathrm{CH}_{3}\right)_{3}\right) ;{ }^{13} \mathrm{C} \mathrm{NMR}$ $\delta_{\mathrm{C}}\left(100 \mathrm{MHz}, \mathrm{CDCl}_{3}\right) 159.4,135.5,132.5,130.0,128.0,75.3,71.7,65.1,61.7,26.9,20.5$, 19.2; m/z (ESI) $399\left(\mathrm{M}^{+}\right)$and $398\left(100 \%, \mathrm{M}-\mathrm{H}^{+}\right)$; HRMS for $\mathrm{C}_{22} \mathrm{H}_{30} \mathrm{NO}_{4} \mathrm{Si}$ requires 400.1944, found 400.1937.

\section{4-(tert-Butyldiphenylsilanyloxy)-1-[2'-(tert-butyldimethylsilanyloxy)-methyl]-but-}

\section{$2 E$-enyl carbamate (5)}
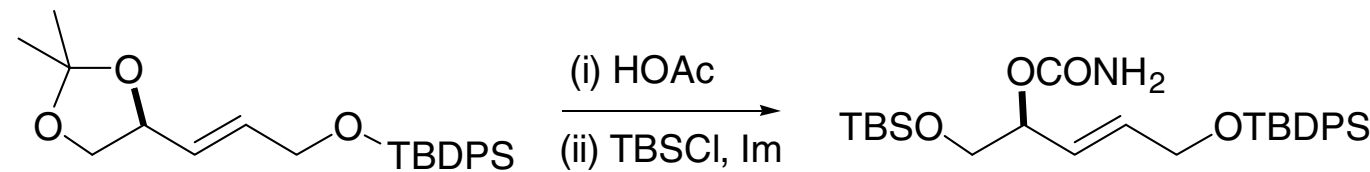

(iii) $\mathrm{Cl}_{3} \mathrm{CCON}=\mathrm{C}=\mathrm{O}$

(tert-Butyldiphenylsilanyloxy)-[3-(2,2-dimethyl-[1,3]dioxolan-4-yl)-allyloxy] ${ }^{5}$ (1.43 g, $3.32 \mathrm{mmol})$ was dissolved in acetic acid $(80 \%, 25 \mathrm{~mL})$ and then warmed to $40^{\circ} \mathrm{C}$ and stirred for $2 \mathrm{~h}$. The mixture was neutralised with $\mathrm{Na}_{2} \mathrm{CO}_{3}$, dissolved in water and extracted with EtOAc (25 mL x4). The crude diol was dissolved in DMF (5 mL) and imidazole (339 mg, $4.98 \mathrm{mmol})$ and tert-butyldimethylchlorosilane (623 mg, $4.15 \mathrm{mmol})$ were added and the reaction stirred at RT for $16 \mathrm{~h}$. The mixture was diluted with water $(50 \mathrm{~mL})$, extracted with EtOAc $(20 \mathrm{~mL} \mathrm{x} 4)$ and the combined organics were washed with brine $(20 \mathrm{~mL})$, dried over $\mathrm{MgSO}_{4}$ and concentrated in vacuo to yield the crude alcohol (24) $(1.26 \mathrm{~g}, 81 \%)$ as a colourless oil. The alcohol was subjected to the standard carbamation conditions to furnish the title compound (5) as a colourless oil (1.06 $\mathrm{g}$, 91\%); ${ }^{1} \mathrm{H}$ NMR $\delta_{\mathrm{H}}\left(200 \mathrm{MHz}, \mathrm{CDCl}_{3}\right)$ 7.70-7.65 (4H, m, Ph), 7.44-7.38 (6H, m, Ph), 5.84-5.82 (2H, m, $\mathrm{C} \underline{\mathrm{H}}=\mathrm{C} \underline{\mathrm{H}}), 5.29-5.21(1 \mathrm{H}, \mathrm{m}, \mathrm{CHOC}), 4.63\left(2 \mathrm{H}\right.$, br s, $\left.\mathrm{NH}_{2}\right), 4.22(2 \mathrm{H}, \mathrm{s}$, 
$\left.\mathrm{CH}_{2} \mathrm{OTBDPS}\right), 3.68\left(2 \mathrm{H}, \mathrm{d}, J\right.$ 5.2, $\left.\mathrm{CH}_{2} \mathrm{OTBS}\right), 1.07\left(9 \mathrm{H}, \mathrm{s},\left(\mathrm{CH}_{3}\right)_{3}\right), 0.91\left(9 \mathrm{H}, \mathrm{s},\left(\mathrm{CH}_{3}\right)_{3}\right)$, $0.09\left(6 \mathrm{H}, \mathrm{s}, \mathrm{Si}\left(\mathrm{CH}_{3}\right)_{2},{ }^{13} \mathrm{C} \mathrm{NMR} \delta_{\mathrm{c}}\left(50 \mathrm{MHz}, \mathrm{CDCl}_{3}\right) 158.1,135.5,133.5,132.6,129.6\right.$, $127.6,124.6,75.4,65.0,63.5,26.8,25.8,19.2,18.3,-5.3 ; \mathrm{m} / \mathrm{z}$ (ESI) 572 ( $100 \%$ $\mathrm{M}+\mathrm{NH}_{4}{ }^{+}+\mathrm{CH}_{3} \mathrm{CN}$ ); HRMS for $\mathrm{C}_{28} \mathrm{H}_{47} \mathrm{~N}_{2} \mathrm{O}_{4} \mathrm{Si}_{2}$ requires 531.3074, found 531.3074.

(1'R,4R,5S)-5-(tert-Butyldimethylsilanyloxy)-4-[2'-(tert-butyldiphenylsilanyloxy)-1'hydroxy-ethyl]-oxazolidin-2-one (6)

Prepared from 4-(tert-butyldiphenylsilanyloxy)-1-[2'-(tert-butyldimethylsilanyloxy)methyl]-but-2E-enyl carbamate (5) via the general TA procedure. The crude material was purified by flash column chromatography on silica, eluting with $40-60^{\circ} \mathrm{C}$ petrol:ethyl acetate (1:1), to furnish the title compound as a colourless oil $(340 \mathrm{mg}, 72 \%, 5: 1) ;[\alpha]^{20}{ }_{\mathrm{D}}$ +32.9 (c. $\left.1.00, \mathrm{CHCl}_{3}\right) ; \mathrm{IR} v_{\max } \mathrm{cm}^{-1}\left(\mathrm{CHCl}_{3}\right) 3379,3073,2938,2834,1743 ;{ }^{1} \mathrm{H} \mathrm{NMR} \delta_{\mathrm{H}}$ (400 MHz, $\left.\mathrm{CDCl}_{3}\right)$ (major isomer) 7.70-7.63 (4H, m, Ph), 7.49-7.40 (6H, m, Ph), 5.51 $(1 \mathrm{H}, \mathrm{br} \mathrm{s}, \mathrm{NH}), 4.39(1 \mathrm{H}, \mathrm{dt}, J$ 8.8, 4.8, CHOCO), $3.87(1 \mathrm{H}, \mathrm{t}, J$ 4.9, CHNH) 3.82-3.62 (4H, m, $\underline{\mathrm{H}}_{2} \mathrm{OTBDPS}$ and $\left.\underline{\mathrm{C}}_{2} \mathrm{OTBDMS}\right), 3.61-3.57(1 \mathrm{H}, \mathrm{m}, \mathrm{C} \underline{\mathrm{HOH}}), 2.84(1 \mathrm{H}, \mathrm{br} \mathrm{s}$, $\mathrm{OH}), 1.10\left(9 \mathrm{H}, \mathrm{s},\left(\mathrm{CH}_{3}\right)_{3}\right), 0.88\left(9 \mathrm{H}, \mathrm{s},\left(\mathrm{CH}_{3}\right)_{3}\right), 0.07\left(6 \mathrm{H}, \mathrm{s}, \mathrm{SiCH}_{3} \mathrm{x} 2\right) ;{ }^{13} \mathrm{C} \mathrm{NMR} \delta_{\mathrm{C}}$ $\left(100 \mathrm{MHz}, \mathrm{CDCl}_{3}\right)$ 158.9, 135.5, 132.3, 130.1, 128.0, 78.3, 71.9, 65.3, 63.1, 56.2, 26.9, 25.7, 19.4, 18.2, -5.5; m/z (ESI) $531\left(100 \%, \mathrm{MH}^{+}\right)$; HRMS for $\mathrm{C}_{28} \mathrm{H}_{43} \mathrm{NO}_{5} \mathrm{Si}_{2}$ requires 530.2758 , found 530.2766 .

\section{(2Z)-1-Propyl-hexenyl carbamate (7)}

(5Z)-Nonen-4-ol ${ }^{6}$ (540 mg, $3.8 \mathrm{mmol}$ ) was subjected to the general carbamate formation procedure, affording the carbamate 7 as an oil $(710 \mathrm{mg}, 100 \%) . R_{\mathrm{f}}(\mathrm{EtOAc}) 0.69$; IR $v_{\max }$ 
(film) 3465, $3350\left(\mathrm{NH}_{2}\right), 2961,2934,2874(\mathrm{CH}), 1713(\mathrm{C}=\mathrm{O})$ and $1600\left(\mathrm{NH}_{2}\right) ;{ }^{1} \mathrm{H}$ NMR $\delta_{\mathrm{H}}\left(400 \mathrm{MHz} ; \mathrm{CDCl}_{3}\right) 5.55(1 \mathrm{H}, \mathrm{dt}, J 10.5$ and $7.5, \mathrm{CH}=\mathrm{C} H \mathrm{Pr}), 5.45(1 \mathrm{H}, \mathrm{dt}, J 8.5$ and 7.5, CHOR), $5.31(1 \mathrm{H}, \mathrm{m}, \mathrm{CHCHOH}), 4.62\left(2 \mathrm{H}, \mathrm{br} \mathrm{s}, \mathrm{NH}_{2}\right), 2.20-2.07(2 \mathrm{H}, \mathrm{m}$, allylic $\left.\mathrm{CH}_{2}\right), 1.73-1.62\left(2 \mathrm{H}, \mathrm{m}, \mathrm{CHOHCH}_{2}\right), 1.54-1.29\left(4 \mathrm{H}, \mathrm{m}\right.$, remaining $\left.\mathrm{CH}_{2}\right), 0.93(3 \mathrm{H}, \mathrm{t}, \mathrm{J}$ 7.5, $\left.\mathrm{CH}_{3}\right)$ and $0.92\left(3 \mathrm{H}, \mathrm{t}, J 7.5, \mathrm{CH}_{3}\right) ;{ }^{13} \mathrm{C} \mathrm{NMR} \delta_{\mathrm{C}}\left(\mathrm{CDCl}_{3}\right) 157.0(\mathrm{C}=\mathrm{O}), 133.7\left(C_{\mathrm{A}}=\mathrm{C}_{\mathrm{B}}\right)$, $128.5\left(\mathrm{C}_{\mathrm{A}}=C_{\mathrm{B}}\right), 70.9(\mathrm{CHOR}), 37.1\left(\mathrm{CH}_{2} \mathrm{CHOR}\right), 29.8,22.6,18.3\left(3 \times \mathrm{CH}_{2}\right), 13.6$ and $13.5\left(2 \times \mathrm{CH}_{3}\right) ; \mathrm{MS} \mathrm{m} / z(\mathrm{CI}) 203\left(\mathrm{MNH}_{4}^{+}\right), 142\left(\mathrm{MH}^{+}-\mathrm{CONH}_{2}\right), 124\left(100 \%, \mathrm{MH}^{+}-\right.$ $\mathrm{OCONH}_{2}$ ) and 95; $\mathrm{HRMS}(\mathrm{CI})$ Found $\mathrm{MNH}_{4}^{+}, 203.1750 . \mathrm{C}_{10} \mathrm{H}_{23} \mathrm{~N}_{2} \mathrm{O}_{2}$ requires $M$ 203.1760.

\section{(1'RS,4RS,5RS)-4-(1'-Hydroxybutyl)-5-propyloxazolidin-2-one (8)}

Prepared from (2Z)-1-propyl-hexenyl carbamate (7) via the general TA procedure. The crude material was purified by flash column chromatography on silica, eluting with ethyl acetate (100\%), to furnish the title compound as a colourless oil (150 mg, 67\%, >10:1); IR $v_{\text {max }} \mathrm{cm}^{-1}\left(\mathrm{CHCl}_{3}\right) 3294,2961,2935,2874,1744 ;{ }^{1} \mathrm{H} \mathrm{NMR} \delta_{\mathrm{H}}\left(400 \mathrm{MHz}, \mathrm{CDCl}_{3}\right) 6.93$ $(1 \mathrm{H}, \mathrm{br} \mathrm{s}, \mathrm{NH}), 4.52(1 \mathrm{H}, \mathrm{dt}, J 8.0$ and $J$ ' 4.5, CHOCO), $4.07(1 \mathrm{H}, \mathrm{br} \mathrm{m}, \mathrm{OH}), 3.62(1 \mathrm{H}$, $\mathrm{m}, \mathrm{C} \underline{\mathrm{HOH}}), 3.36(1 \mathrm{H}, \mathrm{dd}, J 4.0$ and $J$ ' 3.2, $\mathrm{C} \underline{\mathrm{HNH}}), 1.66\left(1 \mathrm{H}, \mathrm{m}, 1 \mathrm{xCH}_{2}\right), 1.58-1.20(7 \mathrm{H}$, $\left.\mathrm{m}, 4 \mathrm{xCH}_{2}\right), 0.92\left(3 \mathrm{H}, \mathrm{t}, J 7.0, \mathrm{CH}_{3}\right), 0.89\left(3 \mathrm{H}, \mathrm{t}, J 7.0, \mathrm{CH}_{3}\right) ;{ }^{13} \mathrm{C} \mathrm{NMR} \delta_{\mathrm{C}}(100 \mathrm{MHz}$, $\left.\mathrm{CDCl}_{3}\right)$ 160.6, 77.6, 71.0, 62.3, 37.9, 33.9, 19.0, 17.9, 13.9, 13.7; m/z (CI) $219\left(\mathrm{MNH}_{4}^{+}\right)$ and $202\left(100 \%, \mathrm{MH}^{+}\right)$; HRMS for $\mathrm{C}_{10} \mathrm{H}_{20} \mathrm{NO}_{3}$ requires 202.1443, found 202.1442 .

\section{4-(tert-Butyldiphenylsilanyloxy)-1-ethyl-but-2Z-enyl carbamate (9)}

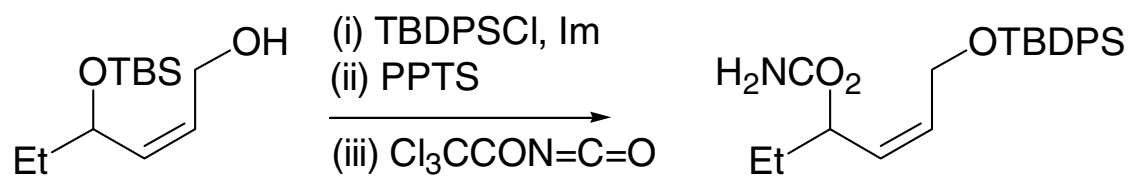


To a stirred solution of 4-(tert-butyldimethylsilanyloxy)-hex-2Z-en-1-ol ${ }^{7}$ (500 mg, 2.17 mmol) in DMF (5 mL) was added imidazole (222 $\mathrm{mg}, 3.26 \mathrm{mmol})$ and tertbutyldiphenylchlorosilane $(0.72 \mathrm{~mL}, 2.74 \mathrm{mmol})$ and the mixture was stirred for $16 \mathrm{~h}$ at RT. The reaction was diluted with water $(50 \mathrm{~mL})$, extracted with ether $(25 \mathrm{~mL} \times 3)$ and the combined organics were washed with brine $(25 \mathrm{~mL})$, dried over $\mathrm{MgSO}_{4}$ and concentrated in vacuo to furnish crude material $(812 \mathrm{mg}, 80 \%)$ as a colourless oil. This material was dissolved in EtOH $(20 \mathrm{~mL})$ and PPTS was added $(427 \mathrm{mg}, 0.17 \mathrm{mmol})$ and the resultant mixture heated at $55^{\circ} \mathrm{C}$ for $10 \mathrm{~h}$. The reaction was cooled to RT, the solvent concentrated in vacuo and the residue filtered through a pad of silica, eluting with EtOAc to furnish the alcohol (453 mg, 77\%) as a colourless oil. The alcohol was subjected to the standard carbamation conditions to furnish the title compound (9) (365 mg, 98\%) as a colourless oil; ${ }^{1} \mathrm{H}$ NMR $\delta_{\mathrm{H}}\left(400 \mathrm{MHz}, \mathrm{CDCl}_{3}\right)$ 7.69-7.67 (4H, m, Ph), 7.43-7.36 (6H, m, $\mathrm{Ph}), 5.75\left(1 \mathrm{H}, \mathrm{dt}, J 5.9 J^{\prime}\right.$ 0.7, TBDPSOCH$\left.{ }_{2} \mathrm{C}=\mathrm{CH}\right), 5.35\left(1 \mathrm{H}, \mathrm{dt}, J 8.9 J^{\prime} 2.2\right.$, TBDPSOCH $\left.\mathrm{CH}_{2}=\mathrm{C} \underline{\mathrm{H}}\right), 5.17-5.15(1 \mathrm{H}, \mathrm{m}, \mathrm{CHOC}), 4.53\left(2 \mathrm{H}\right.$, br s, $\left.\mathrm{NH}_{2}\right), 4.39-4.35(2 \mathrm{H}$, m, $\left.\mathrm{CH}_{2} \mathrm{OTBDPS}\right), 1.61\left(1 \mathrm{H}\right.$, app. sept, $J$ 7.1, $\left.\mathrm{CH}_{3} \mathrm{C}_{2}\right), 1.47$ (1H, app. sept, J 7.2, $\left.\mathrm{CH}_{3} \mathrm{CH}_{2}\right), 1.07\left(9 \mathrm{H}, \mathrm{s},\left(\mathrm{CH}_{3}\right)_{3}\right), 0.82\left(3 \mathrm{H}, \mathrm{t}, J 7.5, \mathrm{CH}_{3}\right) ;{ }^{13} \mathrm{C} \mathrm{NMR} \delta_{\mathrm{C}}\left(50 \mathrm{MHz}, \mathrm{CDCl}_{3}\right)$ $156.6,136.0,134.1,132.2,130.1,128.9,128.1,73.0,60.9,28.2,27.2,19.6,9.6 ; \mathrm{m} / \mathrm{z}$ (ESI) $456\left(100 \% \mathrm{M}+\mathrm{CH}_{3} \mathrm{CN}+\mathrm{NH}_{4}^{+}\right), 420\left(32 \%, \mathrm{M}^{+}\right)$; HRMS for $\mathrm{C}_{23} \mathrm{H}_{31} \mathrm{NO}_{3} \mathrm{SiNa}$ requires 420.1971, found 420.1976 . 


\section{(1'RS,4RS,5RS)-4-[2'-(tert-Butyldiphenylsilanyloxy)-1'-hydroxy-ethyl]-5-}

\section{ethyloxazolidin-2-one (10)}

Prepared from 4-(tert-butyldiphenylsilanyloxy)-1-ethyl-but-2Z-enyl carbamate (9) via the general TA procedure. The crude material was purified by flash column chromatography on silica, eluting with $40-60^{\circ} \mathrm{C}$ petrol:ethyl acetate $(1: 1)$, to furnish the title compound as a colourless oil $(220 \mathrm{mg}, 74 \%,>10: 1)$; IR $v_{\max } \mathrm{cm}^{-1}\left(\mathrm{CHCl}_{3}\right) 3398,2948,2854,1740 ;{ }^{1} \mathrm{H}$ NMR $\delta_{\mathrm{H}}\left(400 \mathrm{MHz}, \mathrm{CDCl}_{3}\right)$ (major isomer) 7.69-7.64 (4H, m, Ph), 7.48-7.39 (6H, m,

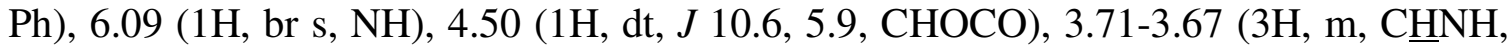
$\left.\mathrm{CH}_{2} \mathrm{OTBDPS}\right) 3.55(1 \mathrm{H}, \mathrm{t}, J 4.4, \mathrm{C} \underline{\mathrm{HOH}}), 3.21(1 \mathrm{H}, \mathrm{d}, J$ 6.5, OH), $1.69(2 \mathrm{H}, \mathrm{dt}, J$ 13.9, 6.9, $\left.\mathrm{CH}_{3} \mathrm{CH}_{2}\right), 1.11\left(9 \mathrm{H}, \mathrm{s},\left(\mathrm{CH}_{3}\right)_{3}\right), 0.97\left(3 \mathrm{H}, \mathrm{t}, J\right.$ 7.3, $\left.\mathrm{CH}_{3}\right) ;{ }^{13} \mathrm{C} \mathrm{NMR} \delta_{\mathrm{C}}(100 \mathrm{MHz}$, $\left.\mathrm{CDCl}_{3}\right) 159.8,135.5,132.6,130.1,127.9,79.9,72.2,64.0,58.4,28.4,26.9,19.2,8.8 ; \mathrm{m} / \mathrm{z}$ (ESI) $414\left(9 \%, \mathrm{MH}^{+}\right), 356(16 \%), 336$ (100\%); HRMS for $\mathrm{C}_{23} \mathrm{H}_{32} \mathrm{NO}_{4} \mathrm{Si}$ requires 414.2078, found 414.2101.

\section{1-(1'-Methylene-propyl)-pentyl carbamate (11)}

3-Methylene-octan-4-ol ${ }^{8}$ (340 $\mathrm{mg}, 2.4 \mathrm{mmol}$ ) was subjected to the general carbamate formation procedure, affording the carbamate $\mathbf{1 1}$ as a low-melting white solid (400 $\mathrm{mg}$, 90\%). $R_{\mathrm{f}}(\mathrm{EtOAc})$ 0.28; IR $v_{\max }\left(\mathrm{CHCl}_{3}\right) 3437,3337\left(\mathrm{NH}_{2}\right), 2958,2936,2874(\mathrm{CH}), 1689$ $(\mathrm{C}=\mathrm{O})$ and $1611\left(\mathrm{NH}_{2}\right) ;{ }^{1} \mathrm{H} \mathrm{NMR} \delta_{\mathrm{H}}\left(400 \mathrm{MHz} ; \mathrm{CDCl}_{3}\right) 5.07(1 \mathrm{H}, \mathrm{t}, J$ 7.0, CHOR $), 5.01$ $\left(1 \mathrm{H}, \mathrm{dt}, J 2.5\right.$ and 1.0, $\left.\mathrm{C}=\mathrm{CH}_{A} \mathrm{H}_{\mathrm{B}}\right), 4.88\left(1 \mathrm{H}, \mathrm{dt}, J 3.0\right.$ and 1.0, $\left.\mathrm{C}=\mathrm{CH}_{\mathrm{A}} H_{B}\right), 4.74(2 \mathrm{H}$, br s, $\left.\mathrm{NH}_{2}\right), 2.05\left(2 \mathrm{H}\right.$, qdd, J 7.5, 1.0 and 1.0, allylic $\left.\mathrm{CH}_{2}\right), 1.66-1.60\left(2 \mathrm{H}, \mathrm{m}, \mathrm{C}^{2} \mathrm{H}_{2}\right), 1.35-$ $1.28\left(4 \mathrm{H}, \mathrm{m}\right.$, remaining $\left.\mathrm{CH}_{2}\right), 1.07\left(3 \mathrm{H}, \mathrm{t}, J 7.5, \mathrm{C}^{3} \mathrm{H}_{3}\right)$ and $0.90\left(3 \mathrm{H}, \mathrm{t}, J 7.0, \mathrm{C}^{5} \mathrm{H}_{3}\right) ;{ }^{13} \mathrm{C}$ NMR $\delta_{\mathrm{C}}\left(\mathrm{CDCl}_{3}\right) 156.5(\mathrm{C}=\mathrm{O}), 149.5\left(C_{\mathrm{A}}=\mathrm{C}_{\mathrm{B}}\right), 109.6\left(\mathrm{C}_{\mathrm{A}}=C_{\mathrm{B}}\right), 77.8(\mathrm{CHOR}), 33.0\left(\mathrm{C}^{2} \mathrm{H}_{2}\right)$, 
$27.5\left(\mathrm{C}^{3} \mathrm{H}_{2}\right), 24.4\left(\right.$ allylic $\left.\mathrm{CH}_{2}\right), 22.5\left(\mathrm{C}^{4} \mathrm{H}_{2}\right), 13.9\left(\mathrm{C}^{5} \mathrm{H}_{3}\right)$ and $12.0\left(\mathrm{CH}_{3}\right) ; \mathrm{MS} m / z(\mathrm{CI}) 203$ $\left(\mathrm{MNH}_{4}^{+}\right), 186\left(\mathrm{MH}^{+}\right), 142\left(\mathrm{MH}^{+}-\mathrm{CONH}_{2}\right), 125\left(100 \%, \mathrm{MH}^{+}-\mathrm{OCONH}_{2}\right)$ and 95; HRMS(CI) Found $\mathrm{MNH}_{4}^{+}$, 203.1768. $\mathrm{C}_{10} \mathrm{H}_{23} \mathrm{~N}_{2} \mathrm{O}_{2}$ requires $M$ 203.1760.

(4RS, 5RS)-5-Butyl-4-ethyl-4-hydroxymethyl-oxazolidinone (12)

Prepared from 1-(1'-methylene-propyl)-pentyl carbamate (11) via the general TA procedure. The crude material was purified by flash column chromatography on silica, eluting with ethyl acetate, to furnish the title compound as a white solid (150 mg, 68\%, 8:1); IR $v_{\max } \mathrm{cm}^{-1}\left(\mathrm{CHCl}_{3}\right) 3288,2957,2874,1732 ;{ }^{1} \mathrm{H} \mathrm{NMR} \delta_{\mathrm{H}}\left(400 \mathrm{MHz}, \mathrm{CDCl}_{3}\right)$ (major isomer) $6.93(1 \mathrm{H}, \mathrm{br} \mathrm{s}, \mathrm{NH}), 4.49(1 \mathrm{H}, \mathrm{dd}, J 6.0,3.0, \mathrm{OH}), 4.20(1 \mathrm{H}, \mathrm{dd}, J$ 6.0, 7.0, CHOCO), $3.62\left(1 \mathrm{H}, \mathrm{dd}, J 12.0,5.0, \mathrm{C}_{\mathrm{A}} \mathrm{CH}_{\mathrm{B}} \mathrm{OH}\right), 3.35\left(1 \mathrm{H}, \mathrm{dd}, J 12.0,7.5, \mathrm{CH}_{\mathrm{A}} \underline{\mathrm{B}}_{\mathrm{B}} \mathrm{OH}\right)$, 1.68-1.43 (5H, m, $\left.\mathrm{CH}_{2}\right), 1.37-1.31\left(3 \mathrm{H}, \mathrm{m}, \mathrm{CH}_{2}\right), 0.91\left(3 \mathrm{H}, \mathrm{t}, J 7.5, \mathrm{CH}_{3}\right), 0.87(3 \mathrm{H}, \mathrm{t}, J$ 7.0, $\left.\mathrm{CH}_{3}\right) ;{ }^{13} \mathrm{C} \mathrm{NMR} \delta_{\mathrm{C}}\left(100 \mathrm{MHz}, \mathrm{CDCl}_{3}\right) 160.2,82.4,64.5,64.1,28.9,28.4,24.1,22.5$, 13.9, 8.1; m/z (CI) $219\left(\mathrm{MNH}_{4} \mathrm{H}^{+}\right), 202$ (100\%); HRMS for $\mathrm{C}_{10} \mathrm{H}_{23} \mathrm{~N}_{2} \mathrm{O}_{3}$ requires 219.1709, found 219.1715.

\section{1-(1'-Methyl-prop-1Z-enyl) carbamate (13)}

To a stirred solution of 2-bromo cis-but-2-ene $(1.5 \mathrm{~mL}, 15 \mathrm{mmol})$ in ether $(10 \mathrm{~mL})$ at $78^{\circ} \mathrm{C}$ was added $t \mathrm{BuLi}(9.0 \mathrm{~mL}, 1.7 \mathrm{M}$ in pentane) over $10 \mathrm{mins}$ and the resultant solution stirred for a further 30 mins. Butyraldehyde $(1.35 \mathrm{~mL}, 23.44 \mathrm{mmol})$ was added and the reaction stirred for $2 \mathrm{~h}$ before being quenched with saturated $\mathrm{NH}_{4} \mathrm{Cl}(10 \mathrm{~mL})$ and allowed to warm to RT. The mixture was extracted with EtOAc $(25 \mathrm{~mL} \mathrm{x} 4)$ and the combined organics washed with brine $(25 \mathrm{~mL})$, dried over $\mathrm{MgSO}_{4}$ and concentrated in vacuo to 
furnish crude alcohol as a colourless oil (834 $\mathrm{mg}, 44 \%)$. The crude alcohol was subjected to the standard carbamation conditions to yield the title compound (13) as a white solid (901 mg, 80.8\%); m.p. 89-90 ${ }^{\circ} \mathrm{C}$; IR $v_{\max } \mathrm{cm}^{-1}\left(\mathrm{CHCl}_{3}\right) 3400,3210,1692 ;{ }^{1} \mathrm{H}$ NMR $\delta_{\mathrm{H}}(400$ $\left.\mathrm{MHz}, \mathrm{CDCl}_{3}\right) 5.48(1 \mathrm{H}, \mathrm{m}, \mathrm{C}=\mathrm{CH}), 5.08\left(2 \mathrm{H}, \mathrm{br} \mathrm{s}, \mathrm{NH}_{2}\right), 4.95(1 \mathrm{H}, \mathrm{t}, J$ 7.1, CHOC), 1.59-1.45 (8H, m, 2x CH $\mathrm{CH}_{3}$ and $\left.\mathrm{CH}_{2}\right), 1.28-1.19\left(2 \mathrm{H}, \mathrm{m}, \mathrm{CH}_{2} \mathrm{CH}_{3}\right), 0.87\left(3 \mathrm{H}, \mathrm{t}, J\right.$ 7.3, $\left.\mathrm{CH}_{3}\right)$;

${ }^{13} \mathrm{C}$ NMR $\delta_{\mathrm{C}}\left(50 \mathrm{MHz}, \mathrm{CDCl}_{3}\right) 157.6,134.4,122.7,80.4,35.3,19.2,14.3,13.4,11.8$.

(1’S,4R,5R)-4-(1-Hydroxyethyl)-4-methyl-5-propyl-oxazolidin-2-one (14)

Prepared from 1-(1'-methyl-prop-1Z-enyl) carbamate (13) via the general TA procedure.

The crude material was purified by flash column chromatography on silica, eluting with $40-60^{\circ} \mathrm{C}$ petrol:ethyl acetate (1:1), to furnish the title compound as colourless crystals (176 mg, 58\%, >10:1); m.p. 93-95 ${ }^{\circ}$; IR $v_{\max } \mathrm{cm}^{-1}\left(\mathrm{CHCl}_{3}\right) 3421,2949,2852,1745 ;{ }^{1} \mathrm{H}$ NMR $\delta_{\mathrm{H}}\left(400 \mathrm{MHz}, \mathrm{CDCl}_{3}\right)$ (major isomer) $6.91(1 \mathrm{H}$, br s, $\mathrm{NH}), 4.36(1 \mathrm{H}, \mathrm{dd}, J$ 10.7, 2.1, CHOCO), $3.96\left(1 \mathrm{H}\right.$, br s, OH), $3.62(1 \mathrm{H}, \mathrm{m}, \mathrm{CHOH}), 1.69-1.53\left(2 \mathrm{H}, \mathrm{m}, \mathrm{CH}_{2}\right), 1.48-1.32$ $\left(2 \mathrm{H}, \mathrm{m}, \mathrm{CH}_{2}\right), 1.12-1.09\left(3 \mathrm{H}, \mathrm{m}, \mathrm{CH}_{3}\right), 1.11\left(3 \mathrm{H}, \mathrm{s}, \mathrm{CH}_{3}\right), 0.92\left(3 \mathrm{H}, \mathrm{t}, J 9.6, \mathrm{CH}_{3}\right) ;{ }^{13} \mathrm{C}$ NMR $\delta_{\mathrm{C}}\left(100 \mathrm{MHz}, \mathrm{CDCl}_{3}\right)$ 159.5, 82.0, 71.9, 64.1, 32.4, 19.9, 17.4, 15.9, 13.8.

\section{1-Cyclohexene-1-yl-pentyl carbamate (15)}<smiles>CCCCC(OC(N)=O)C1=CCCCC1</smiles> 
${ }^{\mathrm{n}} \mathrm{BuLi}(1.6 \mathrm{M}, 3.1 \mathrm{~mL}, 5.0 \mathrm{mmol}$ ) was added dropwise to a stirred solution of 1cyclohexene-1-carboxyaldehyde $(500 \mathrm{mg}, 4.5 \mathrm{mmol})$ in $\mathrm{THF}(20 \mathrm{~mL})$ at $-78{ }^{\circ} \mathrm{C}$ under argon. The solution was allowed to stir at $-78{ }^{\circ} \mathrm{C}$ for 30 minutes before being allowed to warm to room temperature. The reaction was quenched by the addition of water $(5 \mathrm{~mL})$ and diluted with $\mathrm{Et}_{2} \mathrm{O}$. The mixture was washed with aqueous $\mathrm{HCl}(2 \mathrm{M})$ and brine. The organic layer was collected, dried $\left(\mathrm{MgSO}_{4}\right)$, filtered and concentrated in vacuo to afford the alcohol without purification as an oil (730 mg, 95\%).

1-Cyclohex-1-enyl-pentan-1-ol $(680 \mathrm{mg}, 4.1 \mathrm{mmol})$ was subjected to the general carbamate formation procedure, affording the carbamate $\mathbf{1 5}$ as a white solid $(800 \mathrm{mg}$, 94\%). $R_{\mathrm{f}}(1: 1, \mathrm{EtOAc}-$ petrol $)$ 0.68; m.p. 81-84 ${ }^{\circ} \mathrm{C}$; IR $v_{\max }\left(\mathrm{CHCl}_{3}\right) 3429,3330,3262$, $3200\left(\mathrm{NH}_{2}\right), 2929,2858(\mathrm{CH}), 1688(\mathrm{C}=\mathrm{O})$ and $1612\left(\mathrm{NH}_{2}\right) ;{ }^{1} \mathrm{H}$ NMR $\delta_{\mathrm{H}}(400 \mathrm{MHz}$; $\left.\mathrm{CDCl}_{3}\right) 5.69\left(1 \mathrm{H}, \mathrm{td}, J 3.5\right.$ and 1.0, $\left.\mathrm{CH}=\mathrm{CR}_{2}\right), 4.97(1 \mathrm{H}, \mathrm{dd}, J 7.5$ and 6.5, CHOR), 4.73 $\left(2 \mathrm{H}\right.$, br s, $\left.\mathrm{NH}_{2}\right), 2.04-2.02(2 \mathrm{H}, \mathrm{m}), 1.96-1.94(2 \mathrm{H}, \mathrm{m})\left(2 \times\right.$ allylic $\left.\mathrm{CH}_{2}\right), 1.65-1.52(6 \mathrm{H}$, $\mathrm{m}), 1.35-1.22(4 \mathrm{H}, \mathrm{m})$ (remaining $\left.\mathrm{CH}_{2}\right)$ and $0.89\left(3 \mathrm{H}, \mathrm{t}, J \mathrm{~J} .0, \mathrm{CH}_{3}\right) ;{ }^{13} \mathrm{C} \mathrm{NMR}$ $\delta_{\mathrm{C}}\left(\mathrm{CDCl}_{3}\right) 156.7(\mathrm{C}=\mathrm{O}), 136.1\left(\mathrm{CH}=\mathrm{CR}_{2}\right), 124.6\left(C \mathrm{H}=\mathrm{CR}_{2}\right), 79.1(\mathrm{CHOR}), 32.3,27.6$, 24.9, 23.9, 22.5, 22.4 (2 peaks) $\left(7 \times \mathrm{CH}_{2}\right)$ and $13.9\left(\mathrm{CH}_{3}\right) ; \mathrm{MS} m / z(\mathrm{FI}) 211\left(100 \%, \mathrm{M}^{+}\right)$; HRMS (FI) Found $\mathrm{M}^{+}, 211.1578 . \mathrm{C}_{10} \mathrm{H}_{21} \mathrm{NO}_{2}$ requires $M 211.1572$.

\section{(4RS,5SR,6SR)-4-Butyl-6-hydroxy-3-oxa-1-aza-spiro[4.5]decan-2-one (16)}

Prepared from 1-cyclohexene-1-yl-pentyl carbamate (15) via the general TA procedure.

The crude material was purified by flash column chromatography on silica, eluting with ethyl acetate $(100 \%)$, to furnish the title compound as a colourless oil (130 $\mathrm{mg}, 62 \%$, >10:1); IR $v_{\text {max }} \mathrm{cm}^{-1}\left(\mathrm{CHCl}_{3}\right) 3407,3272,2938,2863,1732 ;{ }^{1} \mathrm{H}$ NMR $\delta_{\mathrm{H}}(400 \mathrm{MHz}$, 
$\left.\mathrm{CDCl}_{3}\right)$ (major isomer) $7.55(1 \mathrm{H}, \mathrm{br} \mathrm{s}, \mathrm{NH}), 4.67\left(1 \mathrm{H}, \mathrm{dd}, J 7.0\right.$ and $\left.J^{\prime} 2.5, \mathrm{CHOCO}\right), 3.52$ (1H, br s, OH), $3.24(1 \mathrm{H}$, br m, $\mathrm{C} \underline{\mathrm{HOH}}), 1.90(1 \mathrm{H}, \mathrm{m}), 1.81(1 \mathrm{H}, \mathrm{m}), 1.72(1 \mathrm{H}, \mathrm{m}), 1.64-$ $1.18(11 \mathrm{H}, \mathrm{m}), 0.88\left(3 \mathrm{H}, \mathrm{t}, J 7.5, \mathrm{CH}_{3}\right) ;{ }^{13} \mathrm{C} \mathrm{NMR} \delta_{\mathrm{C}}\left(100 \mathrm{MHz}, \mathrm{CDCl}_{3}\right) 160.4,81.9,71.9$, 64.3, 31.4, 30.7, 29.2, 28.3, 23.8, 22.5, 21.2, 13.9; m/z (CI) $245\left(\mathrm{MNH}_{4}^{+}\right)$and $228(100 \%$, $\mathrm{MH}^{+}$); HRMS for $\mathrm{C}_{12} \mathrm{H}_{25} \mathrm{~N}_{2} \mathrm{O}_{3}$ requires 245.1865, found 245.1861.

\section{1-Vinyl-but-3-enyl carbamate (17)}

1-Vinyl-but-3-enol (1.1 g, $11 \mathrm{mmol}$, commercially available) was subjected to the general carbamate formation procedure, affording the carbamate 17 as an oil $(1.4 \mathrm{~g}, 87 \%) . R_{\mathrm{f}}$ (EtOAc) 0.78; IR $v_{\max }$ (film) 3478, $3351\left(\mathrm{NH}_{2}\right), 3081,3013,2937(\mathrm{CH}), 1722(\mathrm{C}=\mathrm{O})$, $1643(\mathrm{C}=\mathrm{C})$ and $1601\left(\mathrm{NH}_{2}\right) ;{ }^{1} \mathrm{H}$ NMR $\delta_{\mathrm{H}}\left(300 \mathrm{MHz} ; \mathrm{CDCl}_{3}\right)$ 5.86-5.72 $(2 \mathrm{H}, \mathrm{m}), 5.30-$ $5.08(5 \mathrm{H}, \mathrm{m})\left(2 \times \mathrm{CH}=\mathrm{CH}_{2}\right.$ and $\left.\mathrm{CHOCO}\right), 4.74\left(2 \mathrm{H}, \mathrm{br} \mathrm{s}, \mathrm{NH}_{2}\right)$ and $2.41(2 \mathrm{H}, \mathrm{t}, J$ 6.5, $\left.\mathrm{C}^{2} \mathrm{H}_{2}\right) ;{ }^{13} \mathrm{C} \mathrm{NMR} \delta_{\mathrm{C}}\left(\mathrm{CDCl}_{3}\right) 156.1(\mathrm{C}=\mathrm{O}), 136.1,133.1\left(\mathrm{C}^{2}\right.$ and $\left.\mathrm{C}^{5}\right), 117.8,116.4\left(\mathrm{C}^{1}\right.$ and $\left.\mathrm{C}^{6}\right), 74.4\left(\mathrm{C}^{4}\right), 38.8\left(\mathrm{C}^{3}\right)$; MS m/z (CI) $159\left(100 \%, \mathrm{MNH}_{4}^{+}\right)$and $142\left(\mathrm{MH}^{+}\right)$; HRMS (CI) Found $\mathrm{MNH}_{4}^{+}, 159.1136 . \mathrm{C}_{7} \mathrm{H}_{15} \mathrm{~N}_{2} \mathrm{O}_{2}$ requires $M$ 159.1134.

\section{5-(RS)-Allyl-4-(RS)-hydroxymethyl-oxazolidin-2-one (18)}

Prepared from 1-vinyl-but-3-enyl carbamate (17) via the general TA procedure. The crude material was purified by flash column chromatography on silica, eluting with 40$60^{\circ} \mathrm{C}$ petrol-ethyl acetate (1:1), to furnish the title compound as a colourless oil $(450 \mathrm{mg}$, 71\%, 9:1); IR $v_{\text {max }} \mathrm{cm}^{-1}\left(\mathrm{CHCl}_{3}\right) 3334,1735 ;{ }^{1} \mathrm{H} \mathrm{NMR} \delta_{\mathrm{H}}\left(400 \mathrm{MHz}, \mathrm{CDCl}_{3}\right)$ (major isomer) 6.98-6.96 $(1 \mathrm{H}, \mathrm{br} \mathrm{s}, \mathrm{NH}), 5.77\left(1 \mathrm{H}, \mathrm{ddt}, J\right.$ 17.0, 10.5, 7.0, $\left.\mathrm{CH}_{2}=\mathrm{C} \underline{\mathrm{H}}\right), 5.21-5.11$ $\left(2 \mathrm{H}, \mathrm{m}, \mathrm{C}_{2}=\mathrm{CH}\right), 4.61(1 \mathrm{H}$, br s, OH$), 4.43(1 \mathrm{H}, \mathrm{m}, \mathrm{CHOCO}), 3.71-3.55(2 \mathrm{H}, \mathrm{m}$, 
C. $\left.\underline{\mathrm{H}}_{2} \mathrm{OH}\right), 3.50-3.48(1 \mathrm{H}, \mathrm{br} \mathrm{m}, \mathrm{C} \underline{\mathrm{HNH}}), 2.53-2.39\left(2 \mathrm{H}, \mathrm{m}, \mathrm{CH}_{2}\right) ;{ }^{13} \mathrm{C} \mathrm{NMR} \delta_{\mathrm{C}}(100 \mathrm{MHz}$, $\left.\mathrm{CDCl}_{3}\right) 160.3,131.2,119.6,78.0,63.1,58.5,38.7 ; \mathrm{m} / \mathrm{z}(\mathrm{CI}) 172\left(\mathrm{MNH}_{4}^{+}\right)$and 158 $\left(100 \%, \mathrm{MH}^{+}\right)$; HRMS for $\mathrm{C}_{7} \mathrm{H}_{12} \mathrm{NO}_{3}$ requires 158.0817, found 158.0816.

\section{5-(Benzyloxy)-2-(tert-butyldimethylsilanyloxy)-3E-pentenyl-4-(benzyloxy)-2E-}

\section{butenyl carbamate (19)}<smiles>OC(/C=C/CBr)[C@@H](O)/C=C/COCc1ccccc1</smiles>

(i) $\mathrm{CD}$

(ii) $\mathrm{NH}_{4} \mathrm{OH}$

(iii) $\mathrm{TBSCl}$<smiles>NC(=O)OC(/C=C/COc1ccccc1)C(/C=C/COCc1ccccc1)O[Sb]</smiles>

ÖTBS

To a stirred solution of 1,8-bis-benzyloxy-octa-2,6-diene-4,5-diol ${ }^{9}(2.75 \mathrm{~g}, 7.77 \mathrm{mmol})$ in toluene was added 1,1'-carbonyldiimidazole $(2.52 \mathrm{~g}, 15.54 \mathrm{mmol})$ and resulting solution was refluxed for $17 \mathrm{~h}$. The mixture was cooled and diluted with EtOAc $(25 \mathrm{~mL})$, washed sequentially with water $(25 \mathrm{~mL})$ and brine $(25 \mathrm{~mL})$, and further extracted with EtOAc $(25$ $\mathrm{mL}$ x3). The combined organics were dried over $\mathrm{MgSO}_{4}$ and concentrated in vacuo. The material was re-dissolved in IPA $(30 \mathrm{~mL})$ and ammonium hydroxide $(25 \mathrm{~mL})$ was added and the resultant suspension was stirred at RT for $3.5 \mathrm{~h}$. The mixture was diluted with water $(50 \mathrm{~mL})$ and extracted with EtOAc $(25 \mathrm{~mL} \mathrm{x} 4)$. The combined organics were washed sequentially with $\mathrm{HCl}(3 \mathrm{M}, 25 \mathrm{~mL}), \mathrm{NaHCO}_{3}(25 \mathrm{~mL})$ and brine $(25 \mathrm{~mL})$, dried over $\mathrm{MgSO}_{4}$ and concentrated in vacuo to furnish the crude carbamate (31) as a white solid (2.59 g, $6.52 \mathrm{mmol})$. The carbamate $(2.59 \mathrm{~g}, 6.52 \mathrm{mmol})$ was dissolved in DMF $(15 \mathrm{~mL})$ and imidazole $(1.33 \mathrm{~g}, 19.56 \mathrm{mmol})$ and tert butyldimethylsilyl chloride $(2.45 \mathrm{~g}$ $16.30 \mathrm{mmol}$ ) was added. The mixture was stirred at RT for 16 hours and diluted with water $(50 \mathrm{~mL})$ and extracted with EtOAc $(25 \mathrm{~mL} \times 3)$. The combined organics were washed with brine $(25 \mathrm{~mL})$ and concentrated in vacuo. The crude material was purified 
by flash column chromatography on silica, eluting with petrol-EtOAc $(9: 1)$, to furnish the title compound (19) as a colourless oil $(3.12 \mathrm{~g}, 94 \%) ;{ }^{1} \mathrm{H} \mathrm{NMR} \delta_{\mathrm{H}}\left(400 \mathrm{MHz}, \mathrm{CDCl}_{3}\right)$

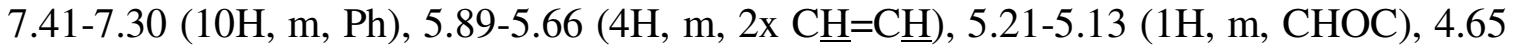
$\left(2 \mathrm{H}\right.$, br s, $\left.\mathrm{NH}_{2}\right), 4.49$ (4H, s, $\left.\underline{\mathrm{C}}_{2} \mathrm{Ph}\right), 4.28$ (1H, app. t, J 5.1, $\left.\mathrm{CH}_{2} \mathrm{OTBDPS}\right), 4.03$ (4H, d, $J$ 4.6, $\left.\mathrm{CH}_{2} \mathrm{OBn}\right), 0.88\left(9 \mathrm{H}, \mathrm{s},\left(\mathrm{CH}_{3}\right)_{3}\right), 0.10\left(3 \mathrm{H}, \mathrm{s}, \mathrm{SiCH}_{3}\right), 0.07\left(3 \mathrm{H} \mathrm{s}, \mathrm{SiCH}_{3}\right) ;{ }^{13} \mathrm{C} \mathrm{NMR}$ $\delta_{\mathrm{C}}\left(50 \mathrm{MHz}, \mathrm{CDCl}_{3}\right) 156.6,138.7,138.6,132.0,130.1,129.5,128.8,128.1,128.0,77.3$, 74.0, 72.7, 72.1, 70.4, 26.2, 18.6, -4.0, -4.4; m/z (ESI) $529\left(100 \% \mathrm{M}+\mathrm{NH}_{4}^{+}\right)$; HRMS for $\mathrm{C}_{29} \mathrm{H}_{45} \mathrm{~N}_{2} \mathrm{O}_{5} \mathrm{Si}$ requires 529.3098, found 529.3088.

\section{$(1 ' R, 5 ' R, 4 R, 5 S)-5-[4 '-B e n z y l o x y-1 '-(t e r t-b u t y l d i m e t h y l-s i l a n y l o x y)-b u t-2 '-e n y l]-4-$}

\section{(6'-benzyloxy-5'-hydroxyethyl)-oxazolidin-2-one (20)}

Prepared from 5-(benzyloxy)-2-(tert-butyldimethylsilanyloxy)-3E-pentenyl-4(benzyloxy)-2E-butenyl carbamate (19) via the general TA procedure. The crude material was purified by flash column chromatography on silica, eluting with $40-60^{\circ} \mathrm{C}$ petrol:ethyl acetate (1:1), to furnish the title compound as a colourless oil (743 $\mathrm{mg}, 72 \%$, $>10: 1) ;[\alpha]^{20}+42.3$ (c. 1.00, $\left.\mathrm{CHCl}_{3}\right) ; \mathrm{IR} v_{\max } \mathrm{cm}^{-1}\left(\mathrm{CHCl}_{3}\right) 3411,3071,2933,2859,1740$, 1598, 1546; ${ }^{1} \mathrm{H}$ NMR $\delta_{\mathrm{H}}\left(400 \mathrm{MHz}, \mathrm{CDCl}_{3}\right)$ 7.39-7.28 (10H, m, Ph), $6.12(1 \mathrm{H}$, br s, NH), $5.90\left(1 \mathrm{H}, \mathrm{dt}, J\right.$ 15.6, $\left.J, 5.5, \mathrm{BnOCH}_{2} \mathrm{C} \underline{\mathrm{H}}=\mathrm{CH}\right), 5.78(1 \mathrm{H}, \mathrm{dd}, J$ 15.6, $J$ ' 5.8, $\left.\mathrm{BnOCH}_{2} \mathrm{CH}=\mathrm{C} \underline{\mathrm{H}}\right), 4.52\left(4 \mathrm{H}, \mathrm{d}, J 8.4,2 \mathrm{xPhC}_{2}\right), 4.46(1 \mathrm{H}, \mathrm{t}, J 4.5, \mathrm{CHOCO}), 4.38(1 \mathrm{H}, \mathrm{t}$, $J$ 5.2, $\left.\mathrm{CH}_{2} \mathrm{OTBDPS}\right), 4.05\left(2 \mathrm{H}, \mathrm{d}, J\right.$ 5.4, $\left.\mathrm{BnOC} \underline{\mathrm{H}}_{2} \mathrm{CH}=\mathrm{CH}\right), 3.76(1 \mathrm{H}, \mathrm{t}, J 3.9, \mathrm{C} \underline{\mathrm{HNH}})$, 3.64-3.51 (3H, m, $\left.\underline{\mathrm{CHOH}}, \underline{\mathrm{C}}_{2} \mathrm{OBn}\right), 3.47\left(1 \mathrm{H}, \mathrm{d}, J\right.$ 7.9, OH), $0.91\left(9 \mathrm{H}, \mathrm{s},\left(\mathrm{CH}_{3}\right)_{3}\right), 0.11$ $\left(6 \mathrm{H}, \mathrm{s}, 2 \mathrm{xSiCH}_{3}\right) ;{ }^{13} \mathrm{C} \mathrm{NMR} \delta_{\mathrm{C}}\left(100 \mathrm{MHz}, \mathrm{CDCl}_{3}\right) 159.3,138.2,137.3,130.8,129.2$, $128.6,128.4,128.0,127.9,127.7,127.6,80.4,73.7,72.3,72.0,71.9,70.9,69.8,55.8$, 
25.8, 18.1, -4.4, -4.9; m/z (ESI) $527\left(\mathrm{M}^{+}\right)$and $526\left(100 \%, \mathrm{M}-\mathrm{H}^{+}\right) ; \mathrm{HRMS}$ for $\mathrm{C}_{29} \mathrm{H}_{42} \mathrm{NO}_{6} \mathrm{Si}$ requires 528.2781, found 528.2773.

\section{References}

1. Mintz, M. J. and Walling, C., Org. Synth., Coll. Vol. V, 1983, 183.

2. Kocovsky, P., Tetrahedron Lett., 1986, 27, 5521

3. Hayashi, T.; Yamamoto, A.; Yoshihiko, I.; Nishioka, E.; Miura, H.; Yanagi, K. J. Am. Chem. Soc., 1989, 111, 6301-6311.

4. Nicolaou, K. C., Seitz, S. P., Pavla, M. R., and Petasis, N. A., J. Org. Chem., 1979, 44, 4011-4013.

5. Cha, J. K., Christ, W. J. and Kishi, Y., Tetrahedron, 1984, 40, 2247-2256.

6. Dittmer, D C.; Discordia, R. P.; Zhang, Y.; Murphy, C. K.; Kumar, A. J.Org.Chem., 1993, 58, 718-731.

7. Gung, B. W., Gerdeman, M. S., Fouch, R. A. and Wolf, M. A., J. Org. Chem., 1994, 59, 4255-4261.

8. Green; M. B.; Hickinbottom; W. J. J. Chem. Soc., 1957; 3262.

9. Takano, S., Kurosaki, A. and Ogasawara, K., Synthesis, 1987, 1075-1078. 